\title{
Chirality-dependent domain wall pinning in a multinotched planar nanowire and chirality preservation using transverse magnetic fields
}

D. S. Eastwood, J. A. King, L. K. Bogart, H. Cramman, and D. Atkinson

Citation: Journal of Applied Physics 109, 013903 (2011);

View online: https://doi.org/10.1063/1.3525733

View Table of Contents: http://aip.scitation.org/toc/jap/109/1

Published by the American Institute of Physics

\section{Articles you may be interested in}

Controlling domain wall pinning in planar nanowires by selecting domain wall type and its application in a memory concept

Applied Physics Letters 92, 022510 (2008); 10.1063/1.2832771

Multimode switching induced by a transverse field in planar magnetic nanowires

Applied Physics Letters 88, 032505 (2006); 10.1063/1.2162263

The design and verification of MuMax3

AIP Advances 4, 107133 (2014); 10.1063/1.4899186

The effect of geometrical confinement and chirality on domain wall pinning behavior in planar nanowires Journal of Applied Physics 104, 033904 (2008); 10.1063/1.2961313

A sound idea: Manipulating domain walls in magnetic nanowires using surface acoustic waves Applied Physics Letters 107, 142405 (2015); 10.1063/1.4932057

Control of the magnetic vortex chirality in Permalloy nanowires with asymmetric notches Journal of Applied Physics 116, 193902 (2014); 10.1063/1.4902008

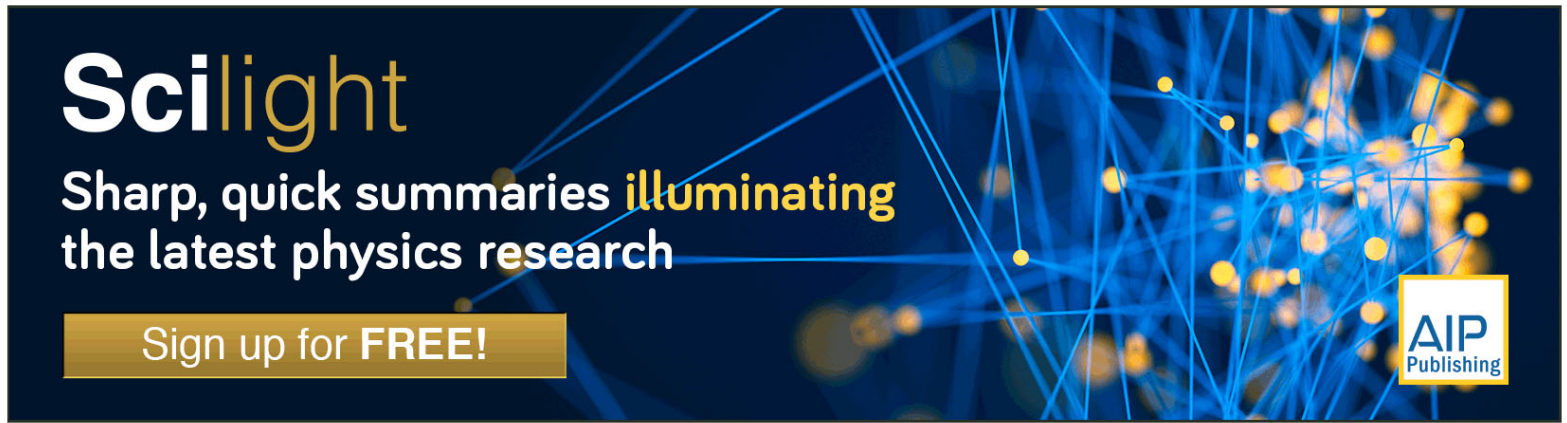




\title{
Chirality-dependent domain wall pinning in a multinotched planar nanowire and chirality preservation using transverse magnetic fields
}

\author{
D. S. Eastwood, ${ }^{\text {a) }}$ J. A. King, L. K. Bogart, H. Cramman, and D. Atkinson \\ Department of Physics, Durham University, South Road, Durham DH1 3LE, United Kingdom
}

(Received 31 August 2010; accepted 9 November 2010; published online 4 January 2011)

Chirality-dependent pinning of magnetic domain walls (DWs) has been observed in a planar $\mathrm{Ni}_{81} \mathrm{Fe}_{19}$ nanowire with two spatially separated notches on opposite edges of the wire. Using focused magneto-optical Kerr effect measurements of field-driven magnetization reversal we show that several different metastable remanent magnetization states can be formed reproducibly, and that the application of a component of magnetic field transverse to the wire axis maintains the chirality of DW as it interacts with both notches and overcomes the problems of chirality loss due to Walker breakdown. (C) 2011 American Institute of Physics. [doi:10.1063/1.3525733]

\section{INTRODUCTION}

Magnetic nanostructures able to retain several remanent magnetization states could be used to store multiple bits of data within the free layer of a single magnetoresistive memory element, potentially allowing increased data storage densities. ${ }^{1}$ It is known that domain walls (DWs) propagating along nanowires experience chirality dependent pinning potentials when notches on one edge of the wire break the axial symmetry, where the chirality is exhibited in the internal structure of the DW. ${ }^{2,3}$ In this paper we show that chirality dependence can be used to set different metastable multidomain configurations at remanence, and we establish the field sequences needed to set each of these states and to ensure that DW chirality is preserved throughout the process.

In a polycrystalline $\mathrm{Ni}_{81} \mathrm{Fe}_{19}$ planar nanowire of quasiinfinite length the lowest energy magnetization state is uniform, lying along the length of the wire axis. When a DW is introduced into the wire (in our study by the reversal of a large "nucleation pad" of magnetic material with weaker shape anisotropy situated at one end of the wire) the total energy increases. Using energy calculations the different DW structures have been mapped onto a phase diagram as a function of wire width and thickness by McMichael and Donahue $^{4}$ and by Nakatani et al. ${ }^{5}$ The two primary types of wall are transverse and vortex. In transverse walls the local magnetic moments between the two axial domains rotate in the plane across the nanowire width. Here the sense of the rotation is labeled as "up" or "down" as shown in Figs. 1(a) and 1(b). For vortex walls the spins form a circulating configuration either clockwise or counterclockwise as in Figs. $1(\mathrm{c})$ and 1(d). Both transverse and vortex walls create magnetic poles that increase the magnetostatic energy but micromagnetic simulations show that vortex walls generally have lower magnetostatic energy than transverse walls in wires of identical dimensions.

In the static zero field state the wire dimensions govern whether a transverse or the vortex wall is most favorable energetically, and for the wires shown in this paper with a

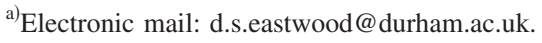

$400 \times 10 \mathrm{~nm}^{2}$ cross-section a vortex wall is expected. ${ }^{5}$ However, when a DW propagates along the wire in a field greater than a threshold $H_{W}$, behavior known as Walker breakdown occurs in which precessional motion of the spins causes stalling and transformation of the DW from a vortex to a transverse wall structure, following which the transverse wall periodically reverses its internal moment via an intermediate vortex or antivortex state. Hayashi et al. ${ }^{8,9}$ demonstrated that this periodic transformation of the wall occurs at discrete multiples of the Larmor precession frequency. This process causes a in DW velocity and changes the chirality of the wall. $^{10-12}$

DW chirality can be selected by applying a small magnetic field transverse to the wire axis (along with the axial field) during DW formation in the nucleation pad. ${ }^{3}$ During propagation DWs with opposite chiralities experience different pinning potentials at structural features on one edge of the wire only. ${ }^{2,3,13-16}$ This is due to the interaction of the spins in the wall as it approaches the spins around the notch which follow the local shape anisotropy; it is seen for triangular notches in experiments and theoretically that if the leading edge of the wall has favorable (near-parallel) alignment to the magnetization immediately after the notch, the potential barrier is low and the wall is likely to propagate past the barrier at or close to the propagation field of the parallel sided wire. ${ }^{7}$ If the spins in the wall lie antiparallel to those after the notch the exchange energy increases when the wall approaches the notch, and a high potential barrier is encountered which requires a large depinning field to be applied before the wall can propagate past the notch. (a)

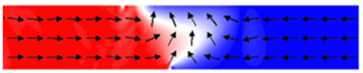

(b)

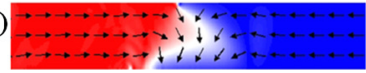

(c)

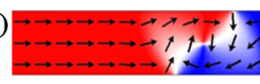

\section{(d)}

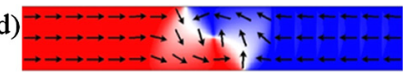

FIG. 1. (Color online) Micromagnetic simulations made using OOMMF (Ref. 6) of (a) an asymmetric transverse DW with a central moment pointing upwards, (b) a downwards asymmetric DW, (c) a clockwise vortex wall, and (d) a counterclockwise vortex wall. Simulations are made for a DW propagating in a $400 \mathrm{~nm}$ wide, $10 \mathrm{~nm}$ thick quasi-infinite length wire. See Ref. 7 for further details of the simulation. 
Importantly, in order to reliably exploit this chirality dependent pinning, chirality flipping due to Walker breakdown must be suppressed. Micromagnetic simulations have previously shown that when the transverse field is aligned parallel to the transverse component of magnetization in a DW Walker breakdown is inhibited and wall velocity increased. ${ }^{17}$ Glathe et al. ${ }^{18}$ performed giant magnetoresistance measurements on multilayer nanowires to experimentally confirm that a transverse field stabilized DW structure and increased DW velocity. ${ }^{19}$ Recently, Weerts et al. ${ }^{20}$ demonstrated suppression of Walker breakdown by superposing a megahertz frequency oscillation upon the axial field. Edge roughness has also been shown to act as a stabilizing factor in micromagnetic simulations. Nakatani et al. ${ }^{11}$ reported that edge roughness with amplitude and wavelength similar to the exchange length caused a dissipation of the precessional motion which causes the antivortex formation and Walker breakdown. A different mechanism for maintaining chirality presented by Lewis et al. ${ }^{21}$ was to use "filters," or periodic protrusions along the length of the wire, which act to reset the DW structure and preserve chirality over distances of several meters.

Reproducibly exploiting chirality-dependent pinning in order to control the position of DWs in multinotched nanowires is discussed in the first part of this paper. In the second section we demonstrate that different magnetization states can be "programmed" using pulses of magnetic field with both axial and transverse components, and that the resulting states are stable at remanence. The final section quantifies the magnitude of the transverse field necessary to inhibit Walker breakdown.

\section{EXPERIMENTAL}

Planar nanowire structures were patterned by exposing a $70 \mathrm{~nm}$ thick polymethyl methacrylate (PMMA) $495 \mathrm{~K}$ resist to a $360 \mu \mathrm{C} \mathrm{cm}^{-2}$ electron beam dose using a FEI XL30SFEG scanning electron microscope (SEM). The resist was developed in one part methyl isobutyl ketone (MIBK) to three parts propan-1-ol, and used as a mask for thermal evaporation of $\mathrm{Ni}_{81} \mathrm{Fe}_{19}$ followed by ultrasonic lift-off.

Here the planar $\mathrm{Ni}_{81} \mathrm{Fe}_{19}$ wires were $100 \mu \mathrm{m}$ long, had a width $390 \pm 15 \mathrm{~nm}$ and thickness $10.0 \pm 0.1 \mathrm{~nm}$ and had two triangular indentations of depth $190 \pm 5 \mathrm{~nm}$ made into opposite sides of the structure at a separation of $40 \mu \mathrm{m}$ (as shown in Fig. 2). Additionally, a large $\left(10 \times 4 \mu \mathrm{m}^{2}\right)$ nucleation pad attached to one end of the wire was designed to reverse at low axial fields, forming a DW at the junction of the pad and the wire which then would "inject" at higher axial field and pass down the length of the wire, through the notches to a tapered annihilation tip. The direction of a field component transverse to the wire axis was used to set the initial chirality of the DW as up or down, and to influence the chirality during propagation.

The axial magnetization was measured using a focused longitudinal magneto-optical Kerr effect (MOKE) system. A linearly polarized $658 \mathrm{~nm}$ focused laser spot was projected at a $45^{\circ}$ angle of incidence onto a $7 \times 5 \mu \mathrm{m}^{2}$ spot on the sample surface. After the reflected beam passed through a

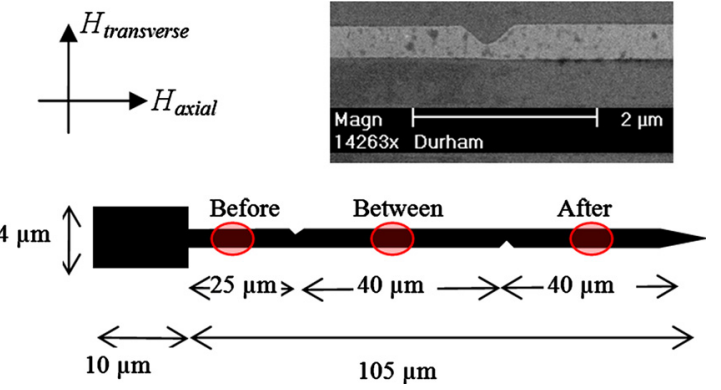

FIG. 2. (Color online) The design of the nanowire (not to scale), and the relation to the axial and transverse field components. The ellipses indicate the three positions where the focused laser spot was placed while taking MOKE measurements. Inset: SEM image of a typical notch in the nanowire. The wire width was measured as $390 \pm 15$ and the notch depth as $190 \pm 5 \mathrm{~nm}$.

quarter wave plate and a second ( $\sim 8^{\circ}$ offset) polarizer the measured intensity of the light was proportional to the Kerr rotation induced by the sample magnetization, and therefore proportional to the local magnetization itself. The axial field was cycled at a frequency of $21.5 \mathrm{~Hz}$, and the measured Kerr signal averaged over approximately 500 hysteresis cycles by a digital oscilloscope.

To obtain minor axial hysteresis loop measurements as a function of the transverse field (in effect, programming the magnetization states), arbitrary computer controlled sequences of axial and transverse magnetic fields were sent to a quadropole magnet powered by two bipolar power supplies. The magnetic field values were measured using a Hall probe attached to the pole pieces of the electromagnet. Figure 2 shows the field axes and the positions where the focused laser MOKE measurements were made on the structure.

\section{RESULTS AND DISCUSSION}

Figure 3 shows local hysteresis loops with the laser spot located in three positions on the wire: before the notches

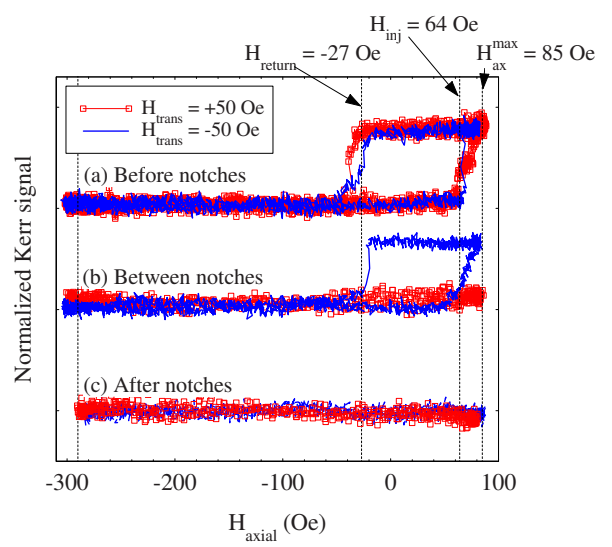

FIG. 3. (Color online) MOKE hysteresis loops from different locations along the nanowire, averaged over 500 hysteresis cycles. The wire is saturated in the negative direction with a large negative axial field, and then partially reversed in the positive direction until the axial field is +90 Oe. $H_{\text {inject }}$ is the axial field at which a DW enters the nanowire from the injection pad. $H_{\text {return }}$ is the lower magnitude, oppositely directed field at which a DW pinned at a notch depins in the reverse direction, suggesting asymmetric pinning at the notch. 


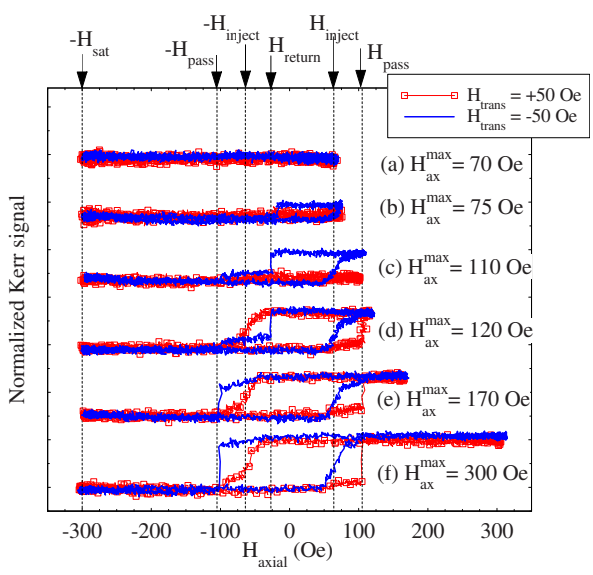

FIG. 4. (Color online) MOKE hysteresis loops measured after one notch but before the second notch. In (a)-(e), local hysteresis loops are taken to an increasing maximum axial field, demonstrating that between 80 and 120 Oe switching occurs if and only if a positive transverse field is also applied. In (f), the major hysteresis loop. Chirality dependent pinning is evident from the different switching fields for DWs in upwards and downwards transverse fields.

(just after the nucleation pad); between the notches; and after the two notches. In each loop a constant transverse field either +50 or -50 Oe was applied to set the DW chirality while the axial field was reversed starting from negative saturation. With $H_{\text {trans }}=-50 \mathrm{Oe}$, the DW passes the first notch at $H_{\mathrm{axial}}^{\mathrm{max}}=85$ Oe but is pinned at the second. With a transverse field of +50 Oe the DW is pinned at the first notch. The measurements after the notch show no magnetization reversal indicating that neither chirality of wall passes both notches demonstrating that chirality flipping does not occur between the notches. When a larger $H_{\text {axial }}^{\max }=120$ Oe was applied (not shown), both chiralities of DW passed both notches, overriding any chirality dependent pinning and confirming the magnetic continuity and integrity of the wire.

The margins between the pinning field values observed for walls with opposite chiralities are examined in more detail in Fig. 4, where the laser spot was placed between the two notches and the field was cycled through minor hysteresis loops with varying $H_{\mathrm{axial}}^{\mathrm{max}}$. When $H_{\mathrm{axial}}^{\max }=+70 \mathrm{Oe}$, neither up nor down chirality of DW passes the first notch. For $H_{\text {axial }}^{\max }=+75$ Oe some down walls passed the first notch but the small change in Kerr signal indicates only a small probability of passage. In loop (c) at $H_{\text {axial }}^{\text {max }}=H_{\text {pass }}=+110$ Oe there was a complete passage probability for walls with a down chirality but zero probability for the opposite chirality walls. At $H_{\mathrm{axial}}^{\mathrm{max}}=+120$ Oe the axial field is sufficient to depin the strongly pinned up DWs, and overcome the chirality dependent pinning.

Interestingly, in loops (b), (c), and (d) the internotch section is seen to reverse at $H_{\text {return }}=-27$ Oe, a much smaller field amplitude than both the injection field for a new DW, and the forward depinning field $H_{\text {pass }}$. At $H_{\text {return }}$ we deduce that DWs pinned at the second notch move back toward the injection pad when the field is reversed. The fact that $\left|H_{\text {return }}\right| \ll\left|H_{\text {pass }}\right|$ suggests that a DW is trapped just before the notch, not symmetrically at the center of the constriction, agreeing with previous observations by Klaui et al. ${ }^{13}$ The

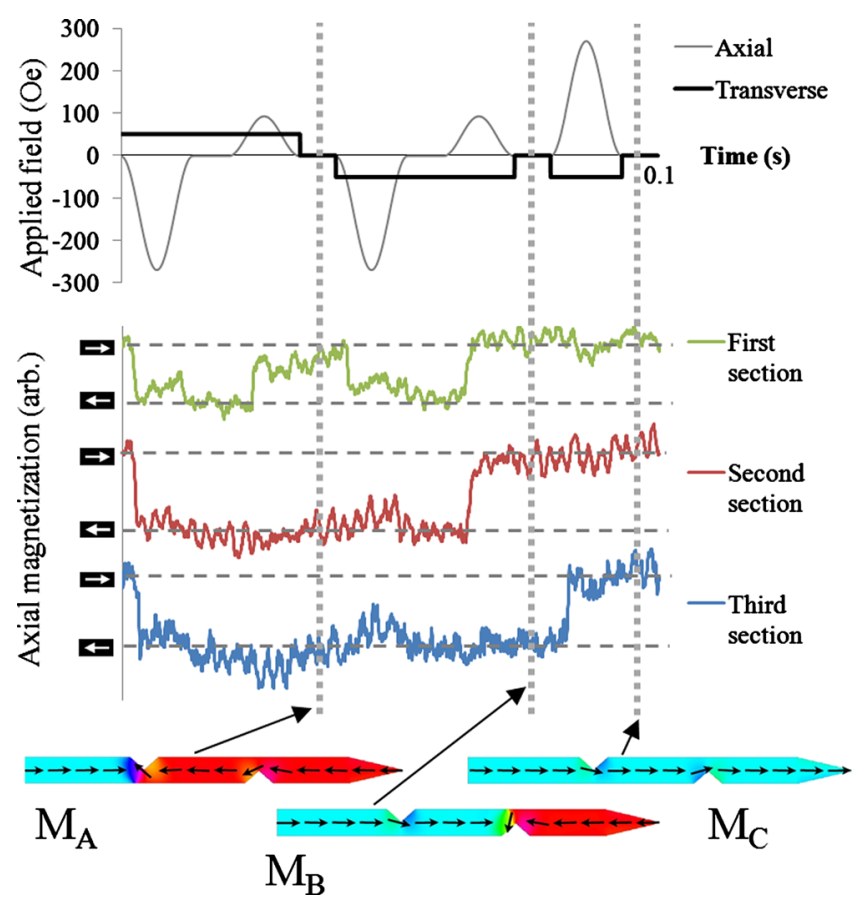

FIG. 5. (Color online) Experimentally applied field components and measurements of the Kerr signal plotted as a function of time, showing three different remanent magnetization states labeled $M_{A}, M_{B}$, and $M_{C}$ and illustrated in schematic magnetization maps excluding the nucleation pad. Measurements are averaged over 700 complete cycles. The actual time period in the experiment was $0.1 \mathrm{~s}$.

absence of the low-field return event in loop (e) suggests that at $H_{\text {axial }}^{\mathrm{max}}=170$ Oe the DW has passed the second notch and been completely expelled from the wire.

The DW behavior inferred from the local hysteresis loops show that a combination of axial and transverse field pulses can selectively pin a DW at either the first or the second notch or reverse the entire length of the nanowire.

In Fig. 5 this control over DW pinning is used to form three different remanent magnetization states in the nanowire, labeled $M_{A}, M_{B}$, and $M_{C}$. The wire was initially saturated in the negative direction, and a small positive axial pulse was applied along the wire in the presence of a positive transverse field. This caused the injection of a DW that was then pinned at the first notch and which remained at remanence $\left(M_{A}\right)$. The wire was then saturated again in the negative direction so that the DW was expelled, and a small axial pulse (this time in a negative transverse field) was sufficient to inject a new DW that passed the first notch and was pinned at the second notch, labeled $M_{B}$. To obtain $M_{C}$ a large positive pulsed field was applied and the DW propagated along the full length of the wire. The equivalent polarityinverted states (which were also measured but are not shown here) provided an additional three distinct magnetization states, giving six possible states in total from a limited combination of axial and transverse fields amplitudes.

The measurement time scale used here was chosen for optimum performance of the MOKE magnetometer; the measurements were effectively quasistatic but the same magnetic functionality is expected in principle over much shorter time scales. The ultimate speed of chirality dependent magnetization reversal is determined by gyroscopic damping of the 


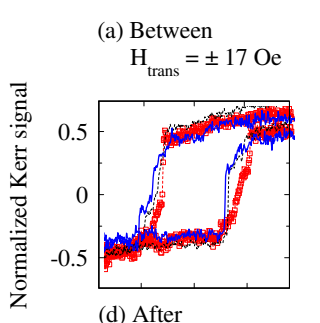

(b) Between
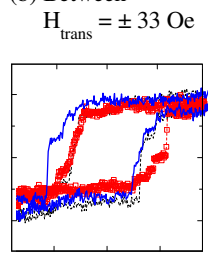

(e) After
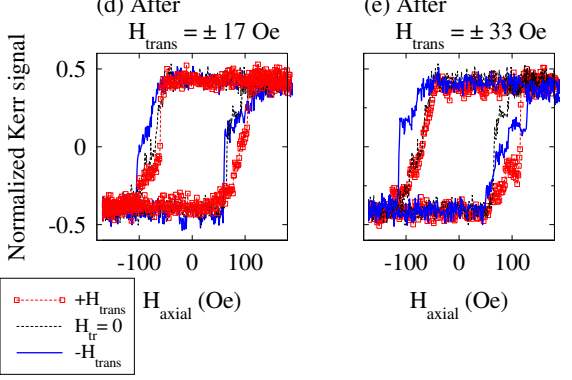

(c) Between $\mathrm{H}_{\text {trans }}= \pm 50 \mathrm{Oe}$

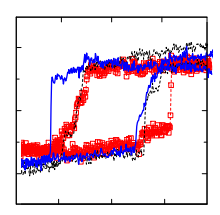

(f) After

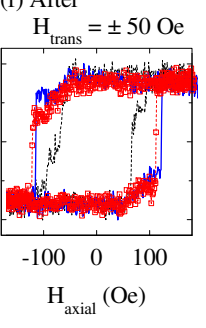

FIG. 6. (Color online) Local hysteresis loops measured by focused MOKE during the application of different transverse fields. Black lines represent a reference zero transverse field measurement, dashed lines with squares represent measurements in positive transverse fields and thick solid lines are in negative transverse fields. [(a)-(c)] Between the notches with $H_{\text {trans }}$ $= \pm 17 \mathrm{Oe}, \pm 33 \mathrm{Oe}$, and $\pm 50 \mathrm{Oe}$, respectively, and $[(\mathrm{d})-(\mathrm{f})]$ after the notches with $H_{\text {trans }}= \pm 17 \mathrm{Oe}, \pm 33 \mathrm{Oe}$, and $\pm 50 \mathrm{Oe}$, respectively. Between the notches a strong chirality dependence is seen for $H_{\text {trans }}= \pm 50$ Oe but this is effect is eliminated after the notches meaning that either up or down walls are pinned at one of the two notches.

spin precession or by the rate at which an applied magnetic field can be varied.

Finally, Fig. 6 shows how the amplitude of the transverse field affects the repeatability of chirality dependent pinning. This figure presents hysteresis loops taken in transverse fields up to +50 Oe (dashed lines) and up to -50 Oe (solid lines), and a reference measurement taken in zero transverse field (solid, black line).

Before the notches both chiralities of DW propagate at approximately 64 Oe irrespective of chirality and transverse field amplitude (as seen earlier in Fig. 3). Figures 6(a)-6(c) show the magnetization between the notches, and Figs. 6(d)-6(f) after the notches, where some degree of chirality dependence is present. For the lower amplitude transverse fields at \pm 17 and \pm 33 Oe in $[(\mathrm{a})$ and (d)] and [(b) and (e)] respectively, any chirality dependence is weak. The hysteresis loops represent an average of 500 magnetization reversals, and the hysteresis curves indicate that individual switching events occur over a spread of axial fields, suggesting subtle variations in DW structure approaching the notch with consequent differences in pinning potentials and also perhaps reflecting some variation due to stochastic thermally activated depinning.

At transverse fields of \pm 50 Oe the switching is sharp indicating a single, repeatable reversal field and a single, specific pinning potential for each chirality. Between the notches the chirality dependent pinning is distinct with walls of compatible chirality with the first notch passing the notch at the injection field, $H_{\text {inject }}$, whereas incompatible walls pass at the higher depinning field, $H_{\text {pass }}$. After both the notches the chirality dependence disappears because all DWs were pinned at one of the two notches, and reversal only occurs at

the higher depinning field $H_{\text {pass }}$. For our wire specifications, this relatively large magnitude of transverse field is needed for reliable chirality dependent pinning.

The absence of significant chirality dependence at low transverse fields [Figs. 6(a) and 6(d)] suggests that the DW structure or chirality at each notch is to some extent random, producing a range of depinning fields. In the larger transverse fields the DW chirality is the same at both notches, and chirality dependent pinning is strong. This is implies one of two effects: either Walker breakdown is completely inhibited by a sufficiently large transverse field (as seen by Bryan et $a l .{ }^{17}$ ), or the chirality of the wall is reset as the DW enters each notch constriction (in an analogous way to the observations of Lewis et al. ${ }^{21}$ ). The second effect is plausible since the passage of a DW through a constriction requires a deformation of the spin structure which increases the exchange energy of the $\mathrm{DW},{ }^{3}$ making a transition to a transverse DW structure more likely than during DW propagation along a wide section of wire. During this transition the chirality could potentially be reset. However any effect only due to structural features would not be expected to depend on the magnitude of the transverse field. Since we observe a strong dependence we propose that the first mechanism is in operation, i.e., that Walker breakdown has been successfully suppressed between the notches by the transverse field.

\section{CONCLUSIONS}

We have shown experimentally that a DW can be selectively pinned at either of two notches in a nanowire, which offers six possible stable magnetization states (when the saturated states and states with reversed magnetic polarity are considered). The field amplitudes needed for reliable chirality dependent pinning have been quantified, and shown to offer sufficient operating margins for highly repeatable writing operation. This writing process may be useful in a multiple-bit magnetic memory layer, which could form the free layer of a magnetoresistive random access memory (MRAM) cell and provide a series of stepped resistance levels through a magnetic tunnel junction. ${ }^{22}$ Storing multiple states within a single memory element may be preferential to using several single-bit elements because strong magnetic interactions can exist between closely-spaced nanomagnetic elements $^{23}$ and nanowires. ${ }^{24}$

Here the extended length of our nanowire was used as it allowed the focused laser spot to be placed between notches for local measurements but the length could be reduced significantly without affecting the selective pinning behavior. Similarly, the time scales over which MOKE observations were made was optimized for the measurement, and the ultimate speed of switching magnetization states is limited either by the gyroscopic spin damping or by the rate at which the applied magnetic field can be pulsed.

The reliability of DW stabilization by a transverse field has been studied. According to the phase diagram given by Nakatani et al..$^{5}$ a wire of the dimensions used here favors vortex DWs in the absence of an applied field or DW motion. However, during motion and under the application of transverse magnetic field component, such walls can be trans- 
formed into transverse walls. ${ }^{17}$ Under a 50 Oe transverse field we observed strong pinning of all DWs at exactly one of the two notches and we are able to confirm that the transverse field either prevents Walker breakdown between the notches, or that the chirality is effectively reset at each notch constriction according to the direction of the transverse field. In either case, a 50 Oe transverse field has been seen to stabilize DW chirality. The local hysteresis loops have shown that a relatively large transverse field is necessary to reliably stabilize DW chirality, and this is in general agreement with the simulations of Bryan et al. ${ }^{17}$

${ }^{1}$ D. Atkinson, D. S. Eastwood, and L. K. Bogart, Appl. Phys. Lett. 92, 022510 (2008).

${ }^{2}$ L. K. Bogart, D. S. Eastwood, and D. Atkinson, J. Appl. Phys. 104, 033904 (2008).

${ }^{3}$ L. K. Bogart, D. Atkinson, K. O'Shea, D. McGrouther, and S. McVitie, Phys. Rev. B 79, 054414 (2009).

${ }^{4}$ R. D. McMichael and M. J. Donahue, IEEE Trans. Magn. 33, 4167 (1997).

${ }^{5}$ Y. Nakatani, A. Thiaville, and J. Miltat, J. Magn. Magn. Mater. 290-291, 750 (2005)

${ }^{6}$ Available at http://math.nist.gov/oommf/.

${ }^{7}$ D. S. Eastwood, L. K. Bogart, and D. Atkinson, Acta Phys. Pol. A 118 719 (2010).

${ }^{8}$ M. Hayashi, L. Thomas, C. Rettner, R. Moriya, and S. S. P. Parkin, Nat. Phys. 3, 21 (2007).

${ }^{9}$ M. Hayashi, L. Thomas, C. Rettner, R. Moriya, and S. S. P. Parkin, Appl.
Phys. Lett. 92, 112510 (2008).

${ }^{10}$ N. L. Schryer and L. R. Walker, J. Appl. Phys. 45, 5406 (1974).

${ }^{11}$ Y. Nakatani, A. Thiaville, and J. Miltat, Nature Mater. 2, 521 (2003).

${ }^{12}$ G. S. D. Beach, C. Nistor, C. Knutson, M. Tsoi, and J. L. Erskine, Nature Mater. 4, 741 (2005).

${ }^{13}$ M. Kläui, H. Ehrke, U. Rudiger, T. Kasama, R. E. Dunin-Borkowski, D. Backes, L. J. Heyderman, C. A. F. Vaz, J. A. C. Bland, G. Faini, E. Cambril, and W. Wernsdorfer, Appl. Phys. Lett. 87, 102509 (2005).

${ }^{14}$ M. T. Bryan, T. Schrefl, and D. A. Allwood, Appl. Phys. Lett. 91, 142502 (2007).

${ }^{15}$ M. Hayashi, L. Thomas, C. Rettner, R. Moriya, X. Jiang, and S. S. P. Parkin, Phys. Rev. Lett. 97, 207205 (2006).

${ }^{16}$ D. Petit, A.-V. Jausovec, D. Read, and R. P. Cowburn, J. Appl. Phys. 103, 114307 (2008)

${ }^{17}$ M. T. Bryan, T. Schrefl, D. Atkinson, and D. A. Allwood, J. Appl. Phys. 103, 073906 (2008)

${ }^{18}$ S. Glathe, R. Mattheis, and D. V. Berkov, Appl. Phys. Lett. 93, 072508 (2008).

${ }^{19}$ S. Glathe, I. Berkov, T. Mikolajick, and R. Mattheis, Appl. Phys. Lett. 93, 162505 (2008).

${ }^{20}$ K. Weerts, W. Van Roy, G. Borghs, and L. Lagae, Appl. Phys. Lett. 96, 062502 (2010)

${ }^{21}$ E. R. Lewis, D. Petit, A. V. Jausovec, L. O’Brien, D. E. Read, H. T. Zeng, and R. P. Cowburn, Phys. Rev. Lett. 102, 057209 (2009).

${ }^{22}$ J. A. King, D. S. Eastwood, L. K. Bogart, H. Armstrong, M. Bath, and D. Atkinson, Technical Proceedings of 2009 NSTI Nanotechnology Conference and Expo, Houston, Texas, 2009, Vol. 1, p. 632.

${ }^{23}$ J. L. Webb and D. Atkinson, J. Appl. Phys. 103, 033905 (2008).

${ }^{24}$ L. O’Brien, D. Petit, H. T. Zeng, E. R. Lewis, J. Sampaio, A. V. Jausovec, D. E. Read, and R. P. Cowburn, Phys. Rev. Lett. 103, 077206 (2009). 